\title{
Sistema Automático para Cultivos Hidropónicos Domésticos, a través de la Medición de Iones Inorgánicos.
}

\section{Automatic System for Domestic Hydroponic Crops, through the Measurement of Inorganic Ions.}

Franklin Geovanni Moreno Montenegro. ${ }^{1}$, Pablo Montejo. ${ }^{2} \&$ Alicia Polanco Risquet. ${ }^{3}$

Recibido: 10-02-2018 / Revisado: 27-02-2019 /Aceptado: 25-03-2019/ Publicado: 05-04-2019

\begin{abstract}
DOI: $\underline{\text { https://doi.org/10.33262/cienciadigital.v9i2.409 }}$

This project refers to the design and implementation of an instrument to monitor in an Android App, the inorganic hydrogen ions, through the measurement of $\mathrm{pH}$, Electroconductivity (EC) and Total Dissolved Solids (TDS), present in the nutritious substance of hydroponic crops and, if necessary, correct the $\mathrm{pH}$ by means of an automatic system.

Free hardware platforms were used, because they were low cost and user friendly. The design is modular due to flexibility, reorganization and construction time.

It consists of five blocks, the first block is an Arduino, responsible for all the control of the system, the second block are sensors that deliver an appropriate signal to Arduino, the third block displays the readings in TFT and stores them in a micro SD, the fourth block has the ability to connect to a Wi-Fi wireless network and through this, access the Web to send the readings to an App on a smartphone, the fifth block controls the dosage of acid or basic substance to balance the value of $\mathrm{pH}$.
\end{abstract}

The read stability of the sensors was checked, the Wi-Fi connection works properly and the readings of these parameters are observed in the cell phone App. It is a device developed with easy-to-purchase components that offers advantages over similar commercial products.

1 Facultad de Informática y Electrónica, Escuela Superior Politécnica de Chimborazo, Chimborazo, Ecuador,fmoreno@espoch.edu.ec

2 Universidad Tecnológica de la Habana José Antonio Echeverría, CUJAE. Centro de Investigaciones en Microelectrónica, Facultad de Ingeniería Eléctrica, La Haba, Cuba, decano.tele@ tesla.cujae.edu.cu

3 Universidad Tecnológica de la Habana José Antonio Echeverría, CUJAE. Centro de Investigaciones en Microelectrónica, Facultad de Ingeniería Eléctrica La Haba, Cuba, alicia.polanco@cime.cujae.edu.cu 
Keywords: Ph, Electroconductivity, Total Dissolved Solids, Hydroponics Control, Arduino, Atlas Scientific, NODEMCU.

\section{Resumen.}

El presente proyecto hace alusión al diseño e implementación de un instrumento para monitorizar en una App de Android, los iones inorgánicos de hidrogeno, a través de la medida de $\mathrm{pH}$, Electroconductividad (EC) y Total de Solidos Disueltos (TDS), presentes en la substancia nutritiva de cultivos hidropónicos y de ser el caso, corregir el pH por medio de un sistema automático.

Se utilizó plataformas de hardware libre, por ser de bajo costo y amigables con el usuario. El diseño es modular por flexibilidad, reorganización y tiempo de construcción.

Consta de cinco bloques, el primer bloque es un Arduino, encargado de todo el control del sistema, el segundo bloque son sensores que entregan una señal apropiada a Arduino, el tercer bloque visualiza las lecturas en TFT y las almacena en una micro SD, el cuarto bloque tiene la capacidad de conectarse a una red inalámbrica Wi-Fi y a través de esta, acceder a la Web para enviar las lecturas a una App en un teléfono inteligente, el quinto bloque controla la dosificación de sustancia acida o básica para equilibrar el valor de $\mathrm{pH}$.

Se comprobó la estabilidad de lectura de los sensores, la conexión Wi-Fi se desempeña adecuadamente y se observan las lecturas de estos parámetros en la App del teléfono celular. Es un dispositivo desarrollado con componentes de fácil adquisición que brinda ventajas sobre productos comerciales similares.

Palabras claves: $\mathrm{Ph}$, Electroconductividad, Total de Solidos Disueltos, Control en Hidroponía, Arduino, Atlas Scientific, NODEMCU.

\section{Introducción.}

La hidroponía es una técnica para cultivar plantas utilizando disoluciones minerales en vez de suelo agrícola. Siguiendo sus principios, las raíces de la planta reciben una solución nutritiva disuelta en agua, con los elementos químicos esenciales para su desarrollo.

Al no exponer la raíz a la contaminación de los suelos, existe mayor limpieza e higiene en el manejo del cultivo, quedando libre de parásitos, bacterias, hongos y contaminación. 
En el Ecuador la hidroponía está en desarrollo y algunas universidades la utilizan como campo de investigación. Pocas empresas privadas están dedicadas a esta actividad, desarrollando métodos de cultivos hidropónicos para la producción de ciertos tipos de vegetales.

Los antecedentes mencionados promueven la incorporación de modernas técnicas de cultivo utilizando herramientas tecnológicas que permitan la medición, el monitoreo y el control de las variables físicas del sustrato nutritivo, para mantenerlo propicio y sostener condiciones idóneas de cultivo.

Estas herramientas tecnológicas deben ser amigables con el usuario, de operación sencilla, con pantalla a colores para destacar cada uno de los parámetros, poseer conectividad para visualizar en un Smartphone los datos que reportan los sensores, además de poder almacenar las lecturas y controlar eventos en el cultivo.

En el mercado existen equipos capaces de medir $\mathrm{pH}$, conductividad eléctrica (EC), total de sólidos disueltos (TDS), poseer alarmas, tener gestión a través de PC, controlar el riego, la fertilización, y monitorización a través de comunicación inalámbrica por vía RF, bluetooth, zigbee, WIFI, GSM, e incluso conectarse a la Web, para almacenar datos y/o monitorear el proceso que implican los cultivos.

Estos equipos electrónicos son una poderosa herramienta de telegestión, pero costosos y están lejos del alcance para algunos usuarios

Por lo antes indicado, el problema científico de esta investigación consiste en la necesidad de desarrollar un equipo electrónico para controlar la calidad de la solución nutriente de cultivos hidropónicos.

De aquí que el objeto de la investigación converge en el estudio de equipos electrónicos con comunicación inalámbrica para garantizar la adecuada calidad del agua de cultivos hidropónicos. El campo de la investigación está enmarcado en la medición de los parámetros en la solución nutriente para cultivos hidropónicos.

El objetivo de esta investigación es diseñar, implementar y validar un prototipo de equipo electrónico con comunicación inalámbrica WiFi que mida el $\mathrm{pH}$, la EC y el TDS, y controle el valor del pH para mantenerlo en el rango de 5.5 a 6.5 que requieren los cultivos hidropónicos.

La presente investigación define como hipótesis: es posible diseñar e implementar un prototipo de equipo electrónico para cultivos hidropónicos con comunicación inalámbrica WiFi, confiable y de bajo costo, basado en una plataforma de hardware libre Arduino y 
sensores para medir el pH, la EC y el TDS, que mediante un actuador controle niveles del $\mathrm{pH}$, muestre en un TFT y en una aplicación para dispositivo móvil, los valores obtenidos por los sensores-

\section{ARQUITECTURA DEL SISTEMA}

La figura 1. Muestra los bloques que conforman el hardware del equipo, se identifica las entradas y las salidas.
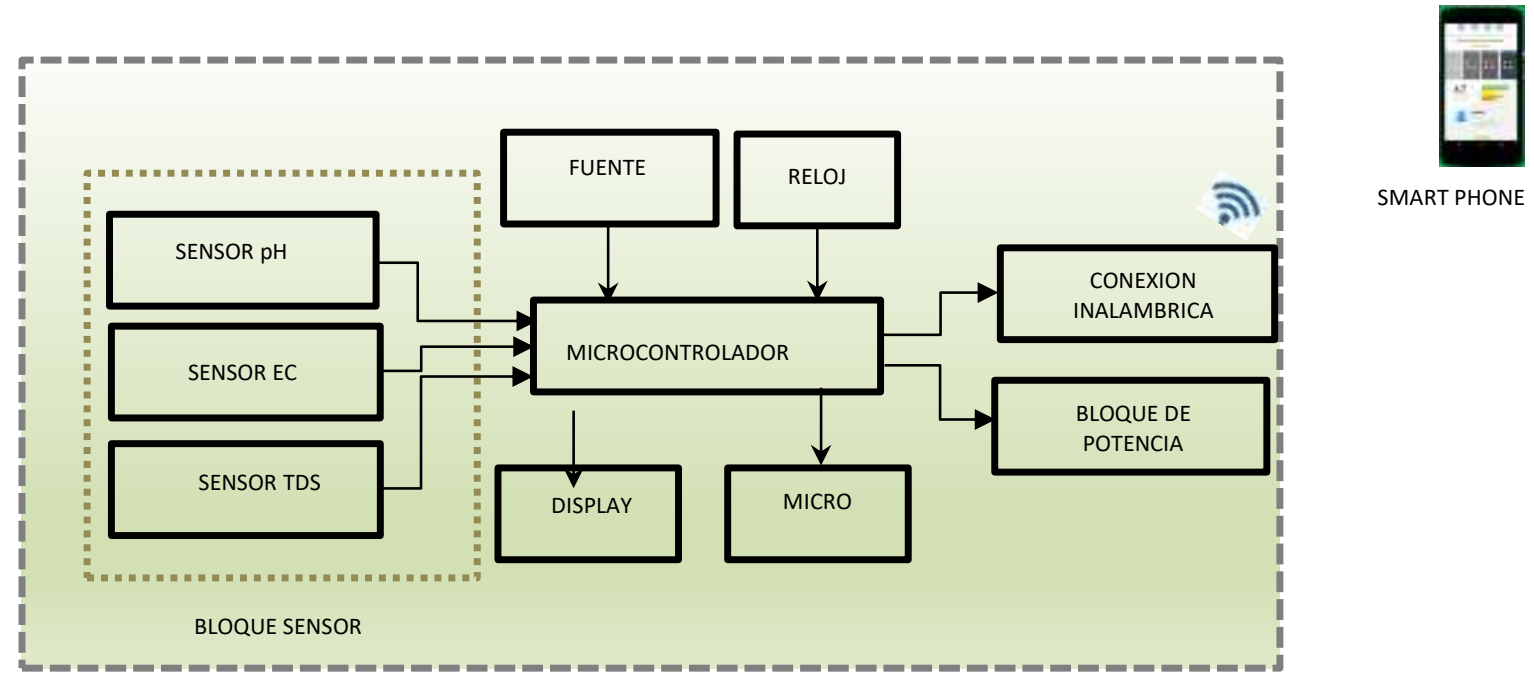

Figura 1.- Diagrama de bloques del equipo electrónico.

Particularicemos la función de cada bloque que conforma el equipo:

- Fuente DC energiza todo el sistema electrónico.

- El bloque MICROPROCESADOR es el encargado de leer la señal proveniente de los sensores, esta lectura se procesa en el software residente en su memoria y se transfiere a los bloques respectivos.

- Bloque de SENSORES está conformado por los sensores de pH, conductividad eléctrica (EC) y total de solidos disueltos (TDS), proporcionan una señal eléctrica en dependencia de la medida de estos parámetros, y la envía al microcontrolador.

- Bloque DISPLAY es el dispositivo donde se visualizan los datos medidos.

- El bloque MICRO SD almacena las lecturas provenientes de los sensores.

- El BLOQUE DE POTENCIA es el encargado de activar un relé para controlar la bomba que añadirá la substancia para nivelar el valor de $\mathrm{pH}$.

- Bloque de CONEXION INALÁMBRICA conecta el equipo a través de WiFi a la WEB para visualizar las lecturas en una aplicación App basada en Android. 
- El bloque RELOJ es un reloj del cual se obtienen la fecha y la hora, esta información será añadida a cada lectura realizada por los sensores.

\section{Elección de dispositivos.}

Para la elección de los dispositivos se considera que sean compatibles con plataformas de código abierto, por su relativa sencillez de programación y sobre todo por la información existente. Se escogieron dispositivos con facilidad de configuración, bajo consumo de energía, baja disipación de potencia y bajo costo.

\section{Funcionalidad de la arquitectura del sistema.}

En la figura 2. Puede observarse él diseño con los respectivos elementos electrónicos escogidos para solventar cada una de las funciones propuestas para el equipo.
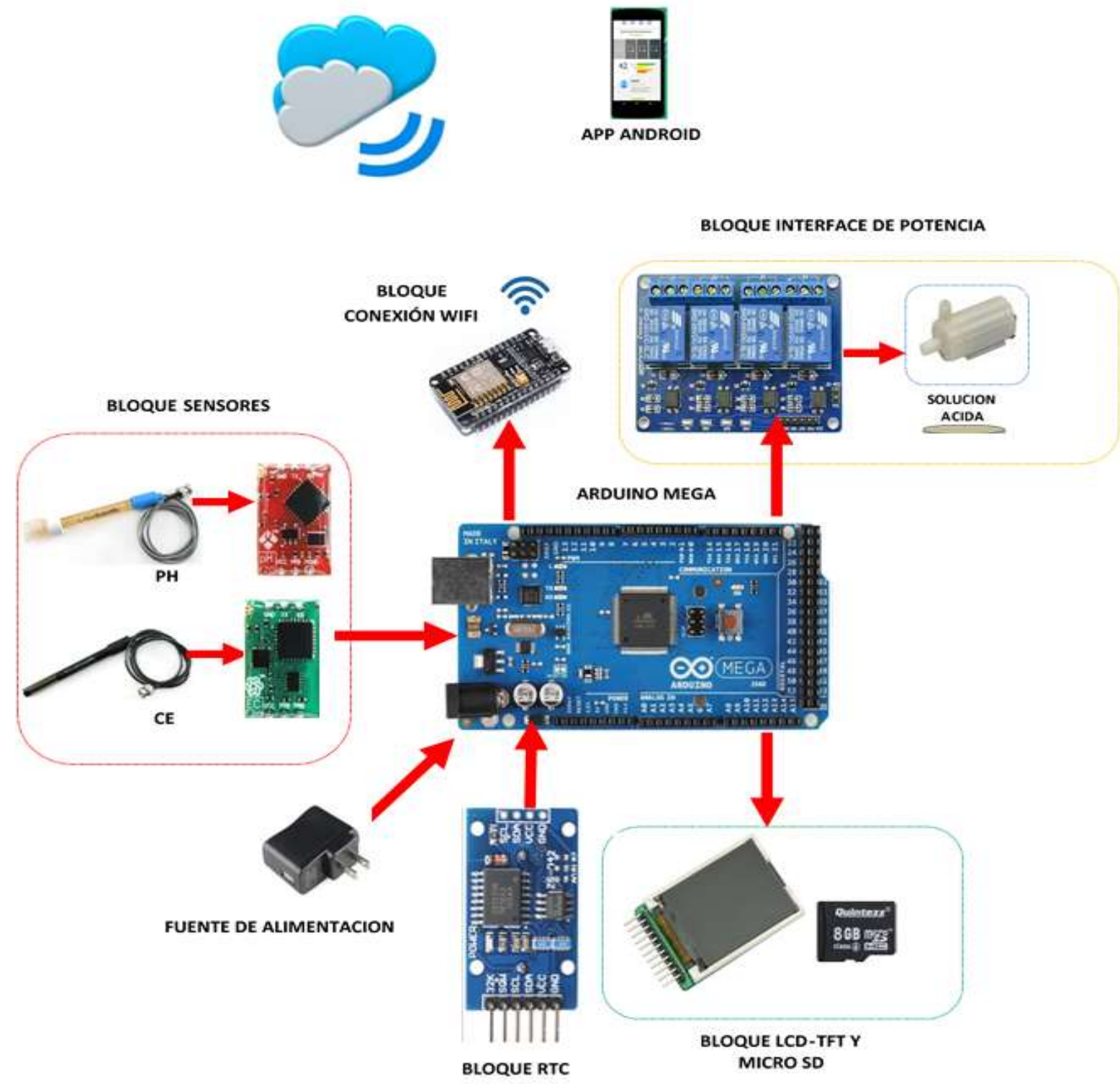

Figura 2.- Diagrama modular del sistema electrónico propuesto. 


\section{Circuito del Prototipo}

Realizado el diseño de la arquitectura por bloques y seleccionados los dispositivos que integran el prototipo, se realizan las conexiones cuyo diagrama se presenta en la figura 3.

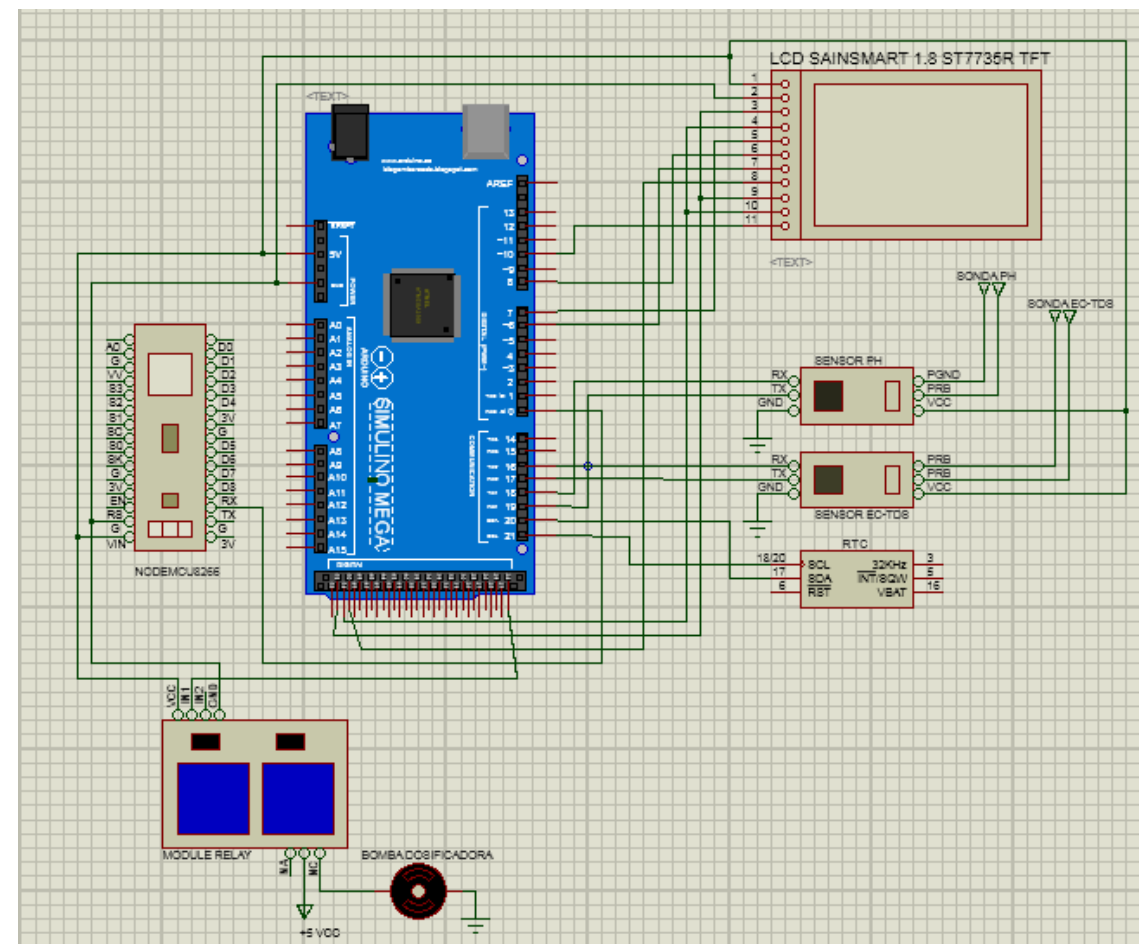

Figura 3.-. Circuito del Prototipo.

\section{DISEÑO DE SOFTWARE}

Se utilizó el entorno de programación de Arduino por la potencialidad que tiene, sus herramientas software y librerías necesarias para configurar y controlar los diferentes dispositivos hardware externos.

Para la monitorización remota se utilizo Blynk que es un creador de aplicaciones para placas de desarrollo como Arduino, ESP8266, y otras plataformas. Nos brinda la posibilidad de construir un tablero digital arrastrando múltiples Widgets interconectados que pueden comunicarse directamente con la placa de desarrollo.

\section{VALIDACIÓN - RESULTADOS}

Para la caracterización del sistema se han utilizado distintos equipos electrónicos comerciales, en nuestro caso dispositivos para la medida de pH y EC-TDS para tener valores de referencia y compararlos con los resultados entregados por el equipo 
implementado. Se realizó las pruebas de almacenamiento de datos en la memoria SD, monitorización vía WIFI, así como la prueba de control del módulo de potencia.

\section{A) Validación de las Medidas de PH - EC - TDS.}

La validación de medidas de $\mathrm{PH}$ entregadas por el prototipo las cotejamos con las mostradas por el Phmetro PH-009(I)A, este mide rangos de $\mathrm{Ph}$ de 0.0 a 14.0 con una resolución de 0.1 y una exactitud de \pm 0.1 ajustándose a las características que deseamos. Para valorar la Electroconductividad y Total de Solidos disueltos, utilizamos como referencia los valores del medidor comercial TDS-EC meter (hold) este posee características que se ajustan a nuestras necesidades: Conductividad: 0-9990 $\mu \mathrm{S} / \mathrm{cm}$, TDS: 0-9990 ppm, Precisión: $\pm 2 \%$.

Las lecturas del sensor de $\mathrm{Ph}$, EC y TDS entregadas por el prototipo y el equipo referencial pueden observarse en la Tabla 1.

Tabla 1. Medición de Ph, EC y TDS con equipo referencial y Prototipo.

\begin{tabular}{|c|c|c|c|c|c|c|c|c|c|}
\hline No & $\begin{array}{c}\text { EC REFERENCIAL } \\
\text { TDS EC meter } \\
\text { (hold) }\end{array}$ & $\begin{array}{c}\text { EC } \\
\text { PROTOTIPO }\end{array}$ & $\begin{array}{c}\text { ERROR } \\
\text { ABSOLUTO }\end{array}$ & $\begin{array}{c}\text { TDS REFERENCIAL } \\
\text { TDS EC meter } \\
\text { (hold) }\end{array}$ & $\begin{array}{c}\text { TDS } \\
\text { PROTOTIPO }\end{array}$ & $\begin{array}{c}\text { ERROR } \\
\text { ABSOLUTO }\end{array}$ & $\begin{array}{c}\text { PH REFERENCIAL } \\
\text { PH-009(I)A }\end{array}$ & $\begin{array}{c}\text { PH PROTOTIPO } \\
\text { ERROR } \\
\text { ABSOLUTO }\end{array}$ \\
\hline 1 & 792 & 740.20 & -51.80 & 396 & 399.70 & 3.7 & 6.9 & 7.17 & 0.27 \\
\hline 2 & 790 & 740.60 & -49.40 & 395 & 399.25 & 4.25 & 6.9 & 7.17 & 0.27 \\
\hline 3 & 790 & 737.21 & -52.79 & 395 & 397.00 & 2.00 & 7.0 & 7.18 & 0.18 \\
\hline 4 & 788 & 734.80 & -53.20 & 394 & 396.80 & 2.80 & 7.0 & 7.17 & 0.17 \\
\hline 5 & 788 & 736.00 & -52.00 & 394 & 397.75 & 3.75 & 7.0 & 7.17 & 0.17 \\
\hline 6 & 788 & 747.21 & -40.79 & 394 & 400.20 & 6.20 & 7.0 & 7.18 & 0.18 \\
\hline 7 & 788 & 741.20 & -46.80 & 394 & 400.20 & 6.20 & 7.0 & 7.17 & 0.17 \\
\hline 8 & 788 & 737.50 & -50.50 & 394 & 398.25 & 4.25 & 7.0 & 7.18 \\
\hline 9 & 788 & 747.80 & -40.20 & 394 & 400.60 & 6.60 & 7.0 & 7.17 \\
\hline 10 & 788 & 740.20 & -47.80 & 394 & 399.70 & 5.70 & 7.0 & 7.18 & 7.17 \\
\hline
\end{tabular}

Se evidencia variación en las lecturas de electroconductividad y total de solidos disueltos, pero al ser medidas en micro Siemens y partes por millón respectivamente, resultan mínimas en relación a sus rangos.

\section{B) Repetitividad en lectura de EC y TDS.}

Este parámetro es de suma importancia y refleja el grado de estabilidad que tienen los sensores, las pruebas se realizaron con los sensores inmersos en la solución química dejándolos reposar unos minutos antes de empezar a tomar las lecturas, estas se observan en la tabla 2. 
Tabla 2 Repetitividad de lecturas.

\begin{tabular}{|c|c|c|c|}
\hline No & PH & $\begin{array}{c}\text { EC } \\
\boldsymbol{\mu S}\end{array}$ & $\begin{array}{c}\text { TDS } \\
\mathbf{p p m}\end{array}$ \\
\hline 1 & 7.15 & 32250 & 17415 \\
\hline 2 & 7.15 & 32250 & 17415 \\
\hline 3 & 7.16 & 32250 & 17418 \\
\hline 4 & 7.15 & 32250 & 17412 \\
\hline 5 & 7.16 & 32250 & 17418 \\
\hline 6 & 7.16 & 32250 & 17416 \\
\hline 7 & 7.18 & 32250 & 17416 \\
\hline 8 & 7.15 & 32250 & 17416 \\
\hline 9 & 7.16 & 32250 & 17417 \\
\hline 10 & 7.16 & 32250 & 17417 \\
\hline $\begin{array}{c}\text { Desviación } \\
\text {-Estándar }\end{array}$ & 0.007 & 3.444 & 2.228 \\
\hline $\begin{array}{c}\text { Media } \\
\text { Coeficiente } \\
\text { de } \\
\text { Variación }\end{array}$ & 7.16 & 32248.70 & 17415.65 \\
\hline
\end{tabular}

Los resultados muestran la estabilidad de los sensores, el coeficiente de variación no llegan al uno por ciento, sin embargo hay que hacer hincapié que dichos valores son tomados pasado algunos minutos. Como puede observarse el error al repetir varias veces la misma medida, es un valor pequeño, además dichos datos están en los rangos esperados.

La figura 4 muestra el Monitor Serial de Arduino, con la repetitividad en las lecturas que entrega el equipo diseñado.

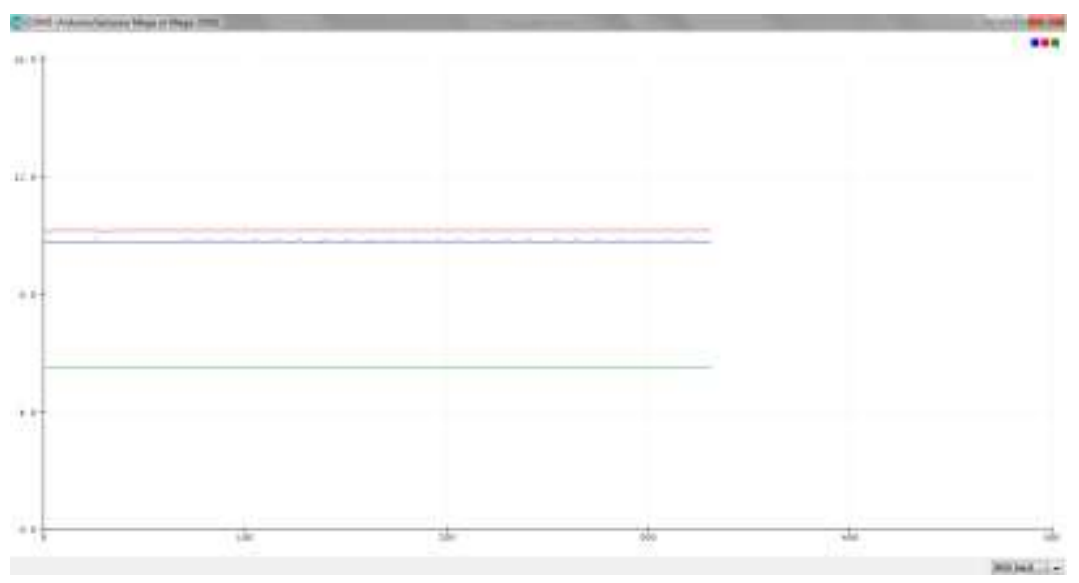

Figura. 4. Repetitividad en las lecturas tomadas desde el monitor serial de Arduino 


\section{C) Pruebas de Almacenamiento en Micro SD}

A través de la programación se creó un archivo del tipo .csv por la facilidad que brinda esta extensión para separar la información en columnas, dicha información contiene los datos de fecha, hora, valor de pH, EC y TDS. La figura 5 muestra el archivo cuyo contenido es la información mencionada. Este archivo puede ser procesado en una hoja de cálculo y se puede procesar según la necesidad de información y/o como datos de investigación.

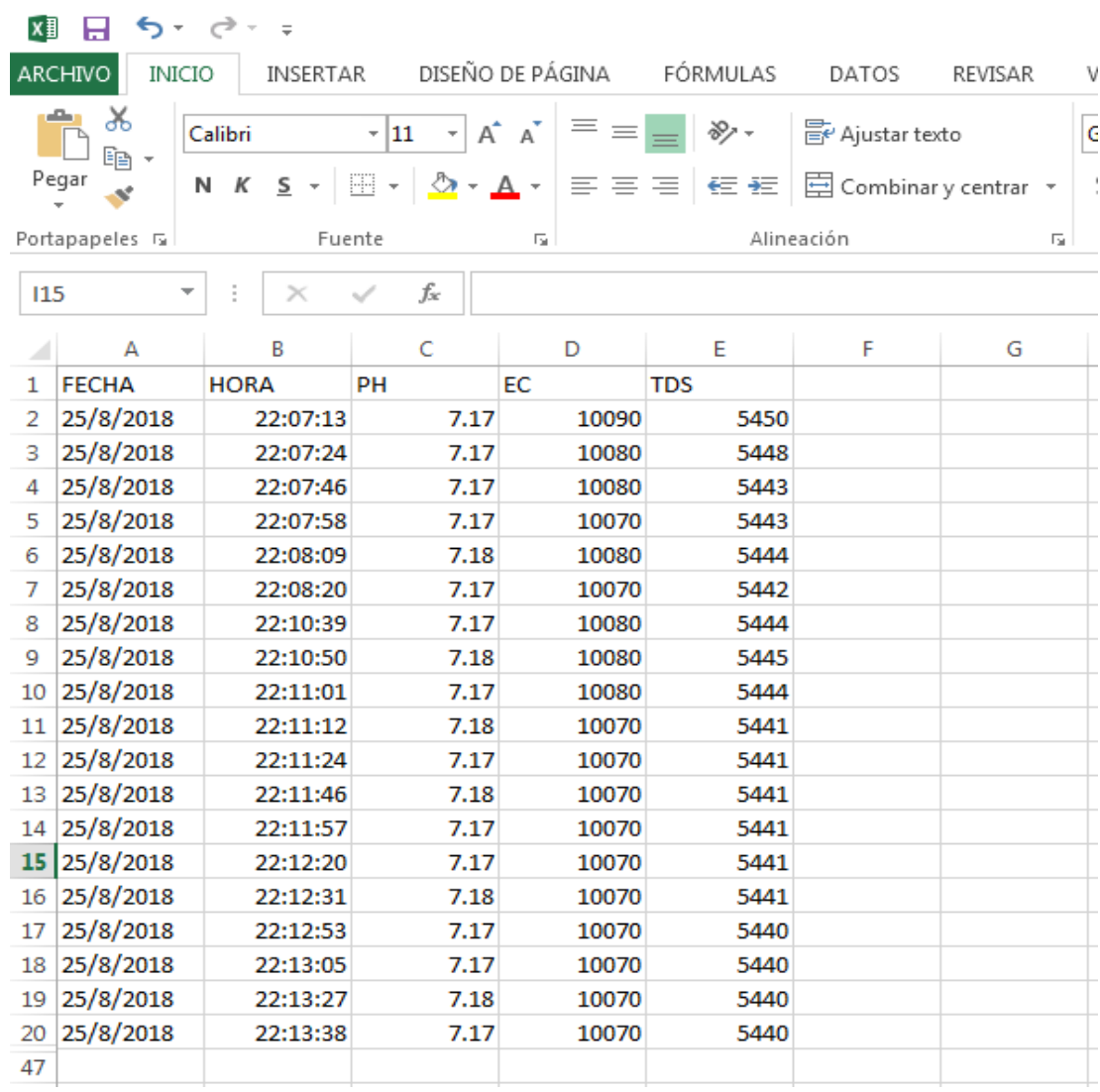

Figura. 5. Archivo generado por el Prototipo

\section{D) Control de Bomba para dosificación.}

Para alcanzar el objetivo de dosificar la substancias acida, se emulo el funcionamiento de una bomba dosificadora a partir de una bomba centrifuga, para inyectar un químico líquido en el seno del fluido hidropónico, para esto se requiere medir el caudal de la bomba.

Se realizaron pruebas para determinar un caudal de la bomba centrifuga, esto se observa en la tabla 3. 
Tabla 3. Medición caudal en bomba centrifuga.

\begin{tabular}{|c|c|c|r|r|r|r|r|}
\hline Volumen & $100 \mathrm{ml}$ & $200 \mathrm{ml}$ & $300 \mathrm{ml}$ & \multicolumn{1}{c|}{$400 \mathrm{ml}$} & $500 \mathrm{ml}$ & $600 \mathrm{ml}$ & $1000 \mathrm{ml}$ \\
\hline & \multicolumn{7}{|c|}{ Tiempo (sg) } \\
\hline 1 & 5.70 & 10.88 & 16.35 & 20.65 & 27.23 & 32.93 & 53.98 \\
\hline 2 & 5.60 & 10.23 & 16.55 & 22.52 & 27.47 & 33.12 & 54.45 \\
\hline 3 & 5.29 & 10.54 & 16.57 & 22.56 & 28.12 & 33.77 & 53.77 \\
\hline 4 & 5.42 & 11.31 & 16.07 & 21.40 & 27.42 & 33.20 & 54.65 \\
\hline 5 & 5.81 & 10.37 & 15.86 & 21.91 & 26.16 & 33.36 & \\
\hline 6 & 5.67 & 10.00 & 16.31 & 21.52 & 27.75 & 32.81 & \\
\hline 7 & 5.68 & 10.13 & 16.27 & 22.12 & 26.97 & 33.12 & \\
\hline 8 & 5.56 & 10.23 & 16.41 & 21.86 & 27.02 & 33.05 & \\
\hline 9 & 5.54 & 10.18 & 16.61 & 21.52 & 26.74 & & \\
\hline 10 & 5.29 & 11.13 & 15.55 & 21.68 & 28.80 & & \\
\hline
\end{tabular}

Delas pruebas anteriores obtenemos:

\begin{tabular}{|c|c|c|c|c|c|c|c|c|c|}
\hline Media & 5.5 & 10.6 & 16.23 & 21.9 & 27.15 & 33.17 & 54.21 & \multirow{2}{*}{5.43} & \multirow{4}{*}{$5.425 \mathrm{sg} / 100 \mathrm{ML}$} \\
\hline Media/100mL & & 5.30 & 5.41 & 5.48 & 5.43 & 5.53 & 5.42 & & \\
\hline Mediana & 5.6 & 10.54 & 16.31 & 21.91 & 27.00 & 33.12 & 54.22 & \multirow{2}{*}{5.42} & \\
\hline Mediana/100mL & & 5.27 & 5.44 & 5.48 & 5.40 & 5.52 & 5.42 & & \\
\hline
\end{tabular}

Se determinó que la bomba cuando es alimentada con 5Vcc alcanza un volumen de 100 $\mathrm{mL}$ en 5,425 sg, a este valor hay que añadirle el tiempo correspondiente en que la substancia recorre la longitud de la manguera hasta alcanzar a la substancia nutritiva. En nuestro caso utilizamos una manguera de $50 \mathrm{~cm}$ de longitud, con lo cual tenemos el tiempo que debe permanecer activada la bomba para llevar la sustancia niveladora de $\mathrm{pH}$.

Cabe destacar que el número de pruebas realizadas supera ampliamente las mostradas en la tabla anterior.

\section{E) Resultados con App.}

Inicialmente se creó un proyecto en la Aplicación Blynk, en este se agregaron los widgets LCD y Serial Terminal para visualizar en el teléfono móvil las lecturas provenientes de los sensores, se configuraron los pines necesarios de la siguiente manera: Serial Terminal el puerto virtual V6 y para LCD el puerto virtual V5. La figura 6 muestra los valores de los parámetros en la aplicación del teléfono celular. 

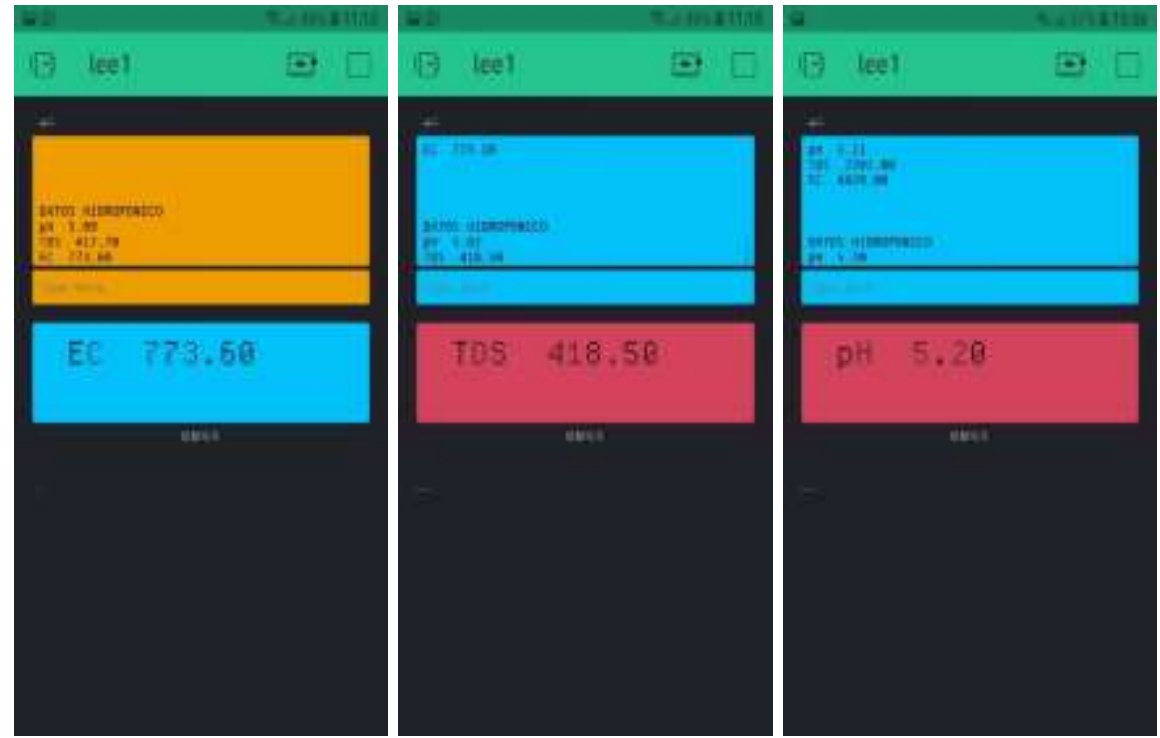

Figura. 6. Datos Recibidos en App de celular

\section{Conclusiones.}

- Se diseñó, implementó y se validó el prototipo de equipo electrónico para Cultivos Hidropónicos Domésticos, a través de la Medición de Iones Inorgánicos, que permite obtener lecturas de pH, EC y TDS, las visualiza en una TFT, almacena las mismas en una micro SD y mediante una conexión WiFi las muestra en una aplicación de teléfono celular, es una herramienta de bajo costo, amigable y útil para las personas dedicadas a cultivos hidropónicos.

- El equipo diseñado mide el pH en un rango de 0 a 14, la electroconductividad de 0 a $200000 \mu \mathrm{S} / \mathrm{cm}$ y el total de solidos disueltos entre 0 y $100000 \mathrm{ppm}$, estos rangos abarcan los valores requeridos para tener una solución nutriente de calidad (el pH entre 5.5 a 6.5 , la electroconductividad de 600 a $4900 \mu \mathrm{S} / \mathrm{cm}$ y el total de solidos disueltos entre 300-2450 ppm)

- De los resultados obtenidos se verificó el adecuado funcionamiento de los sensores de $\mathrm{Ph}, \mathrm{EC}$ y TDS cuyas medidas están dentro de los rangos especificados, la lectura del $\mathrm{pH}$ tiene error relativo a 0.18 correspondiente a $1.3 \%$ en una escala del 0 al 14 , la electroconductividad tiene un error de 49.4 correspondiente a $0.0247 \%$ en una escala entre 5 y 200000 y el total de solidos disueltos tiene un error de 4.4 correspondiente a $0.0247 \%$ en una escala de 2.7 a 108000 . 
- De las pruebas realizadas se comprobó que el equipo entrega lecturas con una desviación estándar pequeña esta indica que la mayor parte de los datos, tienden a estar agrupados cerca de su media aritmética. El coeficiente de variación es pequeño por ende el conjunto de datos es "Homogéneo".

- Se comprobó que el equipo se conecta exitosamente a la Nube a través de WiFi y en la aplicación del celular se visualiza los valores pH, EC y TDS de la solución nutriente.

- Esta arquitectura permite aumentar otros sensores como: oxígeno disuelto (OD), potencial de oxidación-reducción (ORP), iones disueltos de: fluoruro, calcio, magnesio entre otros.

\section{Referencias Bibliográficas.}

J. Beltrano y D. Gimenez.; "Cultivo en hidroponía", Argentina: Editorial de la Universidad de la Plata, 2015.p 10-11

Hydro Environment.; "Calidad del Agua en Hidroponía" . [En línea]. [Citado en: Septiembre del 2018].

https://hydroenv.com.mx/catalogo/index.php?main_page=page \&id=148.

Hydro Environment.; " ¿Qué es una solución nutritiva?" . [En línea]. [Citado en: Septiembre del 2018].

https://hydroenv.com.mx/catalogo/index.php?main_page=page \&id=33

H. Llanos Peada.;"La Solución Nutritiva, Nutrientes Comerciales, Formulas completas", Bogotá, 2001. [En Línea]. [Citado en: septiembre del 2018].

http://www.drcalderonlabs.com/Hidroponicos/Soluciones1.html

Hanna Instruments.; “Medidor multiparámetro con GPS”. [En línea]. [Citado en: Septiembre del

M. Basterrechea.; "Niwa, el sistema hidropónico controlado por smartphone". [En línea]. ( 15 de Junio del 2014). [Citado en: Septiembre del 2018]. https://www.hidroponiacasera.net/niwa-el-sistema-hidroponico-controlado-porsmartphone/ 
Arduino.cl.; “Arduino Mega 2560 R3”. [En Línea]. [Citado en: septiembre del 2018]. http://arduino.cl/Arduino-mega-2560/

Industrial Scientific.; "Sensores electroquímicos". [En Línea]. [Citado en: septiembre del 2018]. http://www.indsci.es/sensores-electroqu\%C3\%ADmicos/

Naylamp mechatronics.; "Módulo RF 433MHz TX y RX”. [En Línea]. [Citado en: septiembre del 2018]. https://naylampmechatronics.com/inalambrico/13-modulo-rf433mhz.html

Adafruit.; "1.8" Color TFT LCD display with MicroSD Card Breakout - ST7735R”. [En Línea].

Dpto. de Física, Ingeniería de Sistemas y Teoría de la Señal.; "Manual de programación de Arduino - Estructura básica de un programa”. [En Línea]. [Citado en: septiembre del 2018]. http://dfists.ua.es/ jpomares/arduino/page_04.htm

GitHub.; “Adafruit-ST7735-Library”. [En Línea]. [Citado en: septiembre del 2018]. https://github.com/adafruit/Adafruit-ST7735-

Library/blob/master/examples/spitftbitmap/spitftbitmap.ino

J. F. García Mejía, J. A. García Mejía, G. A. Gonzáles Hernández y L. A. Tinoco Monroy.; " Obtención de Repetibilidad, Histéresis y Linealidad de un sensor de flexión resistivo por medio de un instrumento virtual". [En Línea]. [Citado en: septiembre del 2018]. http://www.mecamex.net/anterior/cong04/articulos/4CNM-40.PDF

Panamahitek.; "ESP8266 y NodeMCU: la nueva generación de sistemas embebidos”. [En Línea]. [Citado en: septiembre del 2018].

INFOOTEC.NET.; "NodeMCU ESP12”". [En Línea]. [Citado en: septiembre del 2018].

https://www.infootec.net/nodemcu-esp12/\#3--Descripcion-de-cada-uno-de-los-pinesNodeMCU-ESP12

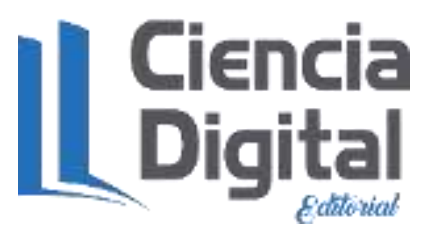




\section{Para citar el artículo indexado.}

Moreno F., Montejo P. \& Polanco A. (2019) Sistema Automático para Cultivos Hidropónicos Domésticos, a través de la Medición de Iones Inorgánicos.

Revista electrónica Ciencia Digital 3(2), 281-291. Recuperado desde: http://cienciadigital.org/revistacienciadigital2/index.php/CienciaDigital/article/view/409/931

\section{Ciencia}

El artículo que se publica es de exclusiva responsabilidad de los autores y no necesariamente reflejan el pensamiento de la Revista Ciencia Digital.

El articulo queda en propiedad de la revista y, por tanto, su publicación parcial y/o total en otro medio tiene que ser autorizado por el director de la Revista Ciencia Digital.
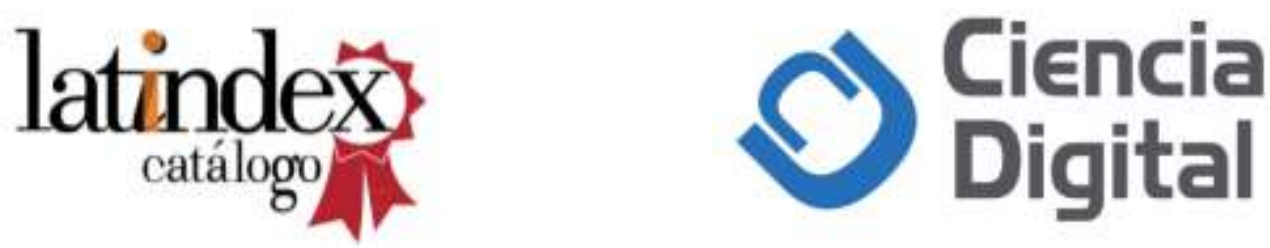